\title{
Evaporative cooling of magnetically trapped atomic molybdenum
}

\section{Citation}

Hancox, Cindy I., Matthew T. Hummon, Scott V. Nguyen, and John M. Doyle. 2005. “Evaporative Cooling of Magnetically Trapped Atomic Molybdenum." Physical Review A 71 (3) (March 11). doi:10.1103/physreva.71.031402.

\section{Published Version}

doi:10.1103/physreva.71.031402

\section{Permanent link}

http://nrs.harvard.edu/urn-3:HUL.InstRepos:28426338

\section{Terms of Use}

This article was downloaded from Harvard University's DASH repository, and is made available under the terms and conditions applicable to Other Posted Material, as set forth at http:// nrs.harvard.edu/urn-3:HUL.InstRepos:dash.current.terms-of-use\#LAA

\section{Share Your Story}

The Harvard community has made this article openly available.

Please share how this access benefits you. Submit a story.

Accessibility 


\title{
Evaporative cooling of magnetically trapped atomic molybdenum
}

\author{
Cindy I. Hancox, Matthew T. Hummon, Scott V. Nguyen, and John M. Doyle \\ Department of Physics, Harvard University, Cambridge, Massachusetts 02138
}

(Dated: October 8, 2004)

\begin{abstract}
We have magnetically trapped and evaporatively cooled atomic molybdenum. Using a cryogenic helium buffer gas, $2 \times 10^{10}$ molybdenum atoms are loaded into a magnetic trap at an initial temperature of $500 \mathrm{mK}$. We measure a molybdenum inelastic rate constant of $g_{\text {in }}=(1.2 \pm 0.6) \times 10^{-12} \mathrm{~cm}^{3} \mathrm{~s}^{-1}$ for the initial conditions after loading. The molybdenum atoms are cooled by adiabatic expansion and forced evaporation to $200 \mathrm{mK}$.
\end{abstract}

PACS numbers: 34.50.-s,32.60.+i, 32.80.Pj

Cooling and trapping is the first step in the creation of ultracold and degenerate quantum gases. Atomic molybdenum has several features that make it an interesting candidate for an ultracold gas. Because of its large magnetic moment (6 Bohr magneton), the dipolar and van der Waals mean-field energies will be similar in a Mo Bose condensate, perhaps leading to new observable dipolar effects [1-5]. Mo has several stable isotopes, 2 fermions and 5 bosons. This opens the possiblity for sympathetic cooling [6-8], of both Fermi and Bose degenerate gases, and interisotope comparisons. In addition, experimental studies of cold, trapped atoms at temperatures above degeneracy can shed light on collision and atomic structure theory [9]. Molybdenum is also important for a variety of studies including double beta decay [10] and biological proteins and enzymes. More detailed knowledge of molybdenum adds to our knowledge base of this element and could one day lead to better understading of the role of Mo in these processes.

The usual route towards magnetic trapping is laser cooling. It is, however, a technique most effective with atoms having energy levels leading to strong transitions at accessible wavelengths. An alternative method is buffer-gas cooling, which is effective in cooling atoms regardless of their internal level structure. In buffergas loading the species-to-be-trapped thermalizes with a cryogenically cooled gas of helium to temperatures below the depth of a magnetic trap.

Once trapped, atoms will undergo collision. Elastic collisions lead to evaporation and cooling of the sample. Inelastic collisions lead to trap loss and limit the efficiency of evaporative. Static magnetic traps confine atoms in high energy, low-field-seeking states. Inelastic spin-changing (i.e. Zeeman-state-changing) collisions lead to trap loss because less magnetic species are preferentially expelled from the trapping region. The dynamics of this loss also leads to heating [11]. It remains an open question as to whether quantum degeneracy can be reached in a magnetic trap with atoms having high magnetic moment as their inelastic spin-relaxation rates will, in general, be higher than lower magnetic moment species (like the alkali metal atoms). So far, only atoms with magnetic moments as high as $2 \mu_{B}$ have been quantum condensed $[12,13]$.

A promising route for reaching degeneracy with high magnetic moment atoms is to evaporatively cool them in a magnetic trap only down to a temperature that is low enough for them to be transferred to a microwave trap $[14,15]$ or far off resonance optical dipole trap (FORT) [16]. In these types of traps they would be confined in the high field seeking, true ground state and evaporative cooling could be continued without inelastic loss. Buffer gas cooling is well suited to the first stage of cooling and loading into a magnetic trap due to the resultant large numbers of trapped atoms.

In this Communication we report trapping of large numbers of Mo atoms, measurement of the inelastic MoMo collision rate constant, and evaporative cooling of Mo. The experimental apparatus is described in detail elsewhere [17]. Briefly, Mo atoms are produced by laser ablation of a metal target inside a cylindrical double-walled plastic cell cooled by a dilution refrigerator (Fig. 1). The jacket formed by the concentric cell walls is filled with superfluid liquid helium that provides a thermal link between the cell and the mixing chamber of the refrigerator. Surrounding the cell is a superconducting anti-Helmholtz magnet which creates a spherical quadrupole trap with a depth of up to $7.2 \mathrm{~K}$. The interior of the cell is filled with a fixed amount of ${ }^{4} \mathrm{He}$ which serves as the buffer gas. The density of buffer gas in the cell is set by the vapor pressure of ${ }^{4} \mathrm{He}$ and can be varied from $<10^{12}$ to $10^{17} \mathrm{~cm}^{-3}$ by heating the cell from 0.2 to $1 \mathrm{~K}$.

The loading procedure begins with a deep $(7.2 \mathrm{~K})$ magnetic trap. The cell is heated to create a high density of buffer gas $\left(10^{17} \mathrm{~cm}^{-3}\right)$ before firing a $15 \mathrm{~mJ}, 5 \mathrm{~ns}$ doubled Nd:YAG (532 nm) ablation pulse onto the Mo target. The target is a piece of natural isotopic abundance molybdenum metal. Roughly $10^{12}$ molybdenum atoms are produced in the ablation. The Mo atoms quickly thermalize with the buffer gas. Low-field-seeking Mo atoms fall into the magnetic trap, while high-field-seeking Mo atoms are driven to the walls where they stick. The cell is cooled to below $0.2 \mathrm{~K}$ within $10 \mathrm{~s}$, causing the buffer gas to liquify onto the cell walls, leaving a magnetically trapped, thermally isolated sample of Mo atoms.

Molybdenum atoms are detected via laser absorption spectroscopy on the $a^{7} S_{3} \rightarrow z^{7} P_{2}$ transition at $390 \mathrm{~nm}$. The $\sim 2 \mathrm{~mm}$ diameter probe beam is produced by passing the output of a Ti:sapphire laser through a resonant 


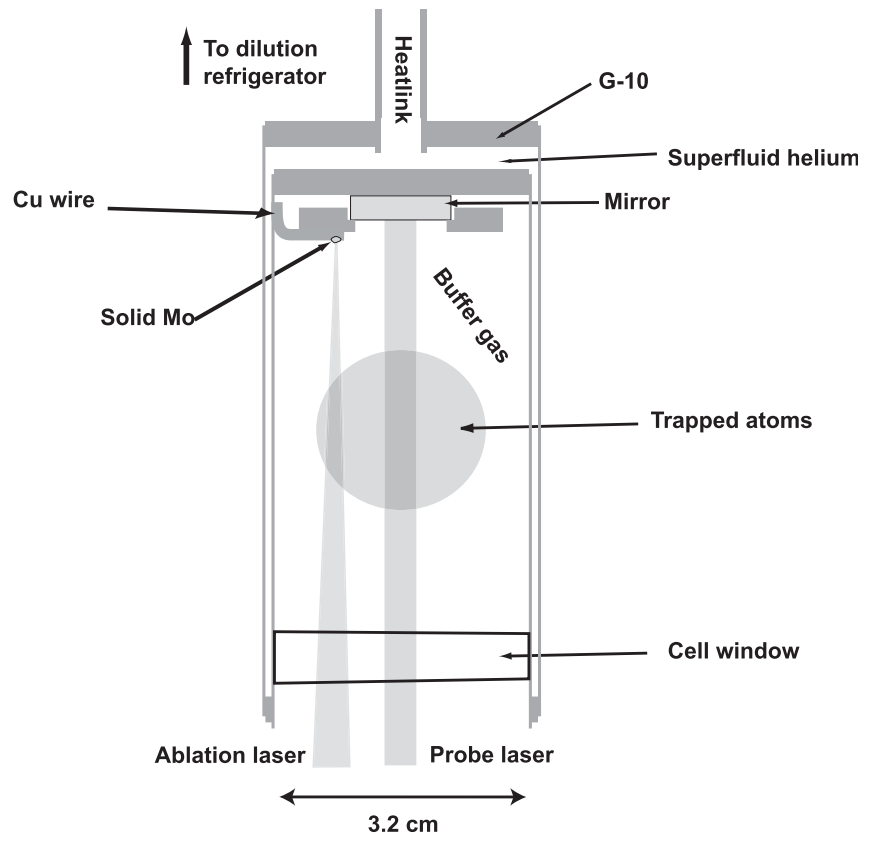

FIG. 1: The experimental plastic cell. The concentric cell walls are filled with superfluid liquid helium that provides thermal conductivity while being electrically insulating.

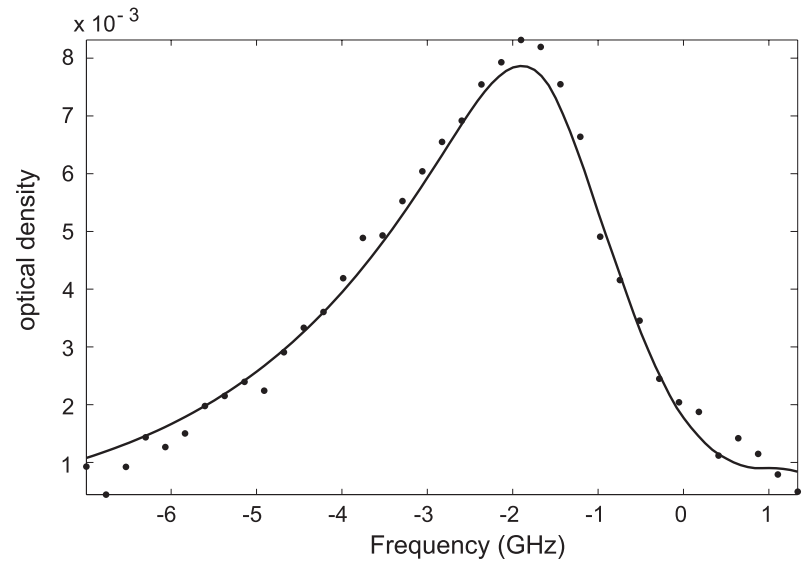

FIG. 2: Absorption spectrum of trapped Mo at the loading trap depth of $7.2 \mathrm{~K}$. The data are shown as filled circles. The solid line is a simulation of a trapped thermal distribution of atoms with number and temperature as free parameters. The fit indicates $2 \times 10^{10}$ Mo atoms trapped at a peak density of $1 \times 10^{11} \mathrm{~cm}^{-3}$ and a temperature of $500 \mathrm{mK}$. The frequency axis contains an arbitrary offset.

doubling cavity. Probe powers of $500 \mathrm{nW}$ are typically used. We verify that the probe light does not affect the loss rate or temperature of the trapped atoms.

Zeeman broadening due to the inhomogeneous trapping field is the dominant broadening mechanism, allowing us to determine the spatial distribution of atoms in the cell from the spectra taken. Fig. 2 shows a spectrum of trapped molybdenum atoms taken at the loading trap depth of $7.2 \mathrm{~K}$ after cryopumping the buffer gas to the

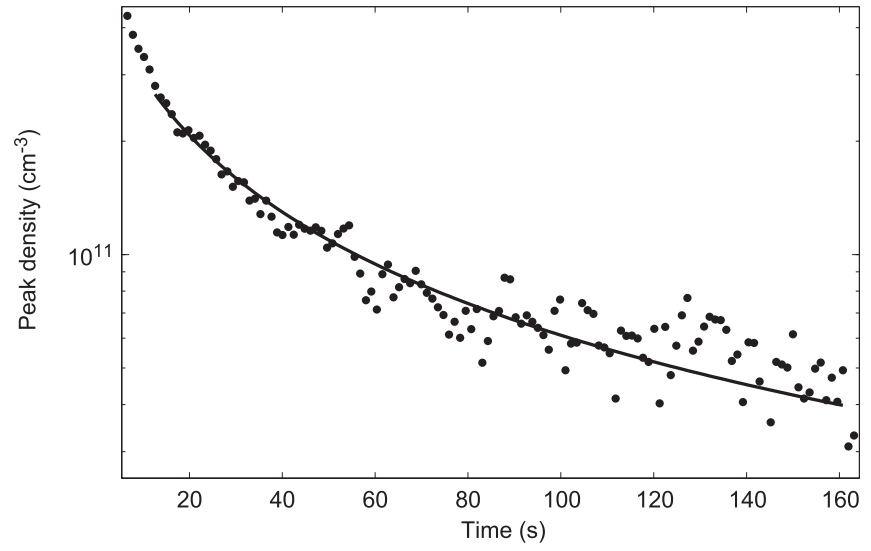

FIG. 3: Peak density as a function of time for trapped Mo atoms. The observed time profile (filled circles) is fit for two body decay due to inelastic collisions, $\mathrm{d} n / \mathrm{d} t=-1 / 8 g_{\mathrm{in}} n^{2}$.

cell walls. The individual isotopes are unresolved due to the large magnetic broadening. A fit to the spectrum finds $(2 \pm 1) \times 10^{10}$ trapped molybdenum atoms at a temperature of $500 \mathrm{mK}$.

We monitor the decay of the trapped atoms to determine the Mo-Mo inelastic collision rate (Fig. 3). The decay is fit to the expected functional form for twobody loss, $\mathrm{d} n / \mathrm{d} t=-1 / 8 g_{\text {in }} n^{2}$, where $g_{\text {in }}$ is the inelastic rate constant, $n$ is the peak atom density in the cell, and the factor of $1 / 8$ is a phase-space factor for our particular trapping geometry [11]. We find $g_{\text {in }}=(1.2+/-0.6) \times 10^{-12} \mathrm{~cm}^{3} \mathrm{~s}^{-1}$. This is similar to the inelastic rate previously seen in $\mathrm{Cr}$, which has the same valence electronic configuration and magnetic moment as Mo [18].

Once the atoms are loaded into the trap, the trapping fields may be reduced to force evaporation. The atoms are cooled both by the evaporation and by adiabatic expansion resulting from the reduced confinement. The electrically insulating plastic cell allows for fast ramping of the magnetic field without inducing eddy currents that would heat the cell. Fig. 4 shows a spectrum taken after ramping the trapping fields to a depth of $0.9 \mathrm{~K}$. The fit shown determines $8 \times 10^{8}$ atoms remaining, cooled to a temperature of $200 \mathrm{mK}$. Atoms are observed exclusively in the fully stretched state $\left(\mid m_{J}=3>\right.$ for the 5 isotopes with zero nuclear spin, $\mid m_{J}=3, m_{I}=5 / 2>$ for the 2 isotopes with $I=5 / 2)$. Although all $m_{J}$ and $m_{I}$ states are produced in the ablation, states other than the fully stretched state are lost to evaporation and spin exchange. Multiple isotopes are resolved; the known isotope shifts and relative natural abundances are used for isotopes with zero nuclear spin [19]. The known isotope shifts and hyperfine constants are used for the positions of the ${ }^{95} \mathrm{Mo}$ and ${ }^{97} \mathrm{Mo}$ peaks [19-21]. One might expect the height of a these peaks to be reduced by a factor of $2 I+1$, however we see a reduction only of a factor of 3 . The fully stretched state population is enhanced after the ablation due to spin exchange, as was seen previously 


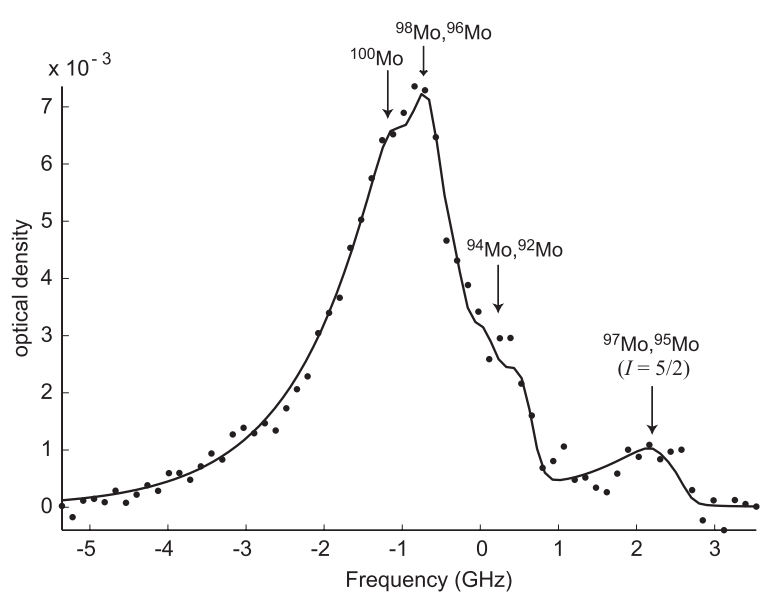

FIG. 4: Absorption spectrum of trapped Mo after forced evaporative cooling. The trapping fields are reduced to a final trap depth of $0.9 \mathrm{~K}$ from the loading depth of $7.2 \mathrm{~K}$. Multiple isotopes are resolved, as indicated.

with $\mathrm{Cr}$ [18]. The trap density is too low to allow for a measurement of the inelastic rate at this temperature.
Detection of further evaporative cooling by lowering the trapping fields was limited by noise on the absorption signal.

In conclusion, we have magnetically trapped all naturally occurring isotopes of molybdenum in large numbers and evaporatively cooled them to a temperature of $200 \mathrm{mK}$. The inelastic Mo-Mo collision rate is measured and found to be similar to that found in $\mathrm{Cr}-\mathrm{Cr}$ collisions. Further cooling of molybdenum to temperatures lower than $200 \mathrm{mK}$ would benefit from a technique such as cooling by evaporation via optical pumping [22], which avoids the decrease in trap densities associated with the lowering of the magnetic trapping field. Another possibility is evaporation by moving the cloud to the surface of the cell [23].

\section{Acknowledgments}

This material is based on work supported by the National Science Foundation under Grant No. PHY0139995. C.I.H. is supported by a National Science Foundation Graduate Research Fellowship.
[1] L. Santos, G. V. Shlyapnikov, P. Zoller, and M. Lewenstein, Phys. Rev. Lett. 85, 1791 (2000).

[2] M. Baranov, L. Dobrek, K. Goral, L. Santos, and M. Lewenstein, Phys. Scr. 74, T102 (2002).

[3] S. Yi and L. You, Phys. Rev. A 66, 013607 (2002).

[4] M. A. Baranov, M. S. Mar'enko, V. S. Rychkov, and G. V. Shlyapnikov, Phys. Rev. A 66, 013606 (2002).

[5] L. Santos, G. V. Shlyapnikov, and M. Lewenstein, Phys. Rev. Lett. 90, 250403 (2003).

[6] A. G. Truscott, K. E. Strecker, W. I. McAlexander, G. B. Partridge, and R. G. Hulet, Science 291, 2570 (2001).

[7] B. DeMarco and D. S. Jin, Science 285, 1703 (1999).

[8] C. J. Myatt, E. A. Burt, R. W. Ghrist, E. A. Cornell, and C. E. Wieman, Phys. Rev. Lett. 78, 586 (97).

[9] C. I. Hancox, S. C. Doret, M. T. Hummon, L. Luo, and J. M. Doyle, Nature. 431, 281 (2004).

[10] P. Doe, H. Ejirii, and S. R. Elliot, Nucl. Phys. A 721, $517 \mathrm{C}(2003)$.

[11] W. Ketterle and N. J. V. Druten, Adv. At. Mol. Opt. Phys. 37, 181 (1996).

[12] A. Robert, O. Sirjean, A. Browaeys, J. Poupard, S. Nowak, D. Boiron, C. I. Westbrook, and A. Aspect, Science 292, 461 (2001).

[13] F. P. Dos Santos, J. Leonard, J. M. Wang, C. J. Barrelet, F. Perales, E. Rasel, C. S. Unnikrishnan, M. Leduc, and
C. Cohen-Tannoudji, Phys. Rev. Lett. 86, 3459 (2001).

[14] I. F. Silvera, C. Gerz, L. S. Goldner, W. D. Phillips, M. W. Reynolds, S. L. Rolston, R. J. C. Spreeuw, and C. I. Westbrook, Phys. B. 194-196, 893 (1994).

[15] C. C. Agosta, I. F. Silvera, H. T. C. Stoof, and B. J. Verhaar, Phys. Rev. Lett. 62, 2361 (1989).

[16] J. D. Miller, R. A. Cline, and D. J. Heinzen, Phys. Rev. A 47, R4567 (1993).

[17] J. Weinstein, Ph.D. thesis, Harvard University (2002).

[18] J. D. Weinstein, R. deCarvalho, C. I. Hancox, and J. Doyle, Phys. Rev. A 65, 021604 (2002).

[19] P. Aufmuth, H.-P. Clieves, K. Heilig, A. Steudel, D. Wendlandt, and J. Bauche, Z. Phys. A 285, 357 (1978).

[20] S. Buttgenbach, M. Herschel, G. Meisel, E. Schrodl, W. Witte, and W. J. Childs, Z. Phys. 266, 271 (1974).

[21] G. Olsson, T. Olsson, L. Robertsson, and A. Rosen, Phys. Scr. 29, 61 (1984).

[22] I. D. Setija, H. G. C. Werij, O. J. Luiten, M. W. Reynolds, T. W. Hijmans, and J. T. M. Walraven, Phys. Rev. Lett. 70, 2257 (1993).

[23] D. M. Harver, J. M. McGuirk, J. M. Obrecht, and E. A. Cornell, J. Low Temp. Phys. 133, 229 (2003). 